\title{
Random Spin-1 Quantum Chains
}

\author{
Beatriz Boechat and Andreia Saguia \\ Instituto de Fisica, Universidade Federal Fluminense \\ Campus da Praia Vermelha, Niteroi, 24210-340, RJ, Brasil \\ Mucio A.Continentino \\ Instituto de Fisica, Universidade Federal Fluminense \\ Campus da Praia Vermelha, Niteroi, 24020-340, Rj, Brasil \\ and \\ National High Magnetic Field Laboratory \\ Florida State University, 1800 E.Paul Dirac Dr. \\ Tallahassee, Fl 32306 USA
}

\begin{abstract}
We study disordered spin-1 quantum chains with random exchange and biquadratic interactions using a real space renormalization group approach. We find that the dimerized phase of the pure biquadratic model is unstable and gives rise to a random singlet phase in the presence of weak disorder. In the Haldane region of the phase diagram we obtain a quite different behavior.
\end{abstract}

KEYWORDS: A. Disordered Systems; D. Phase Transitions, Thermodynamic Properties. 
Although much progress has been made on the understanding of the physical properties of ordered isotropic quantum spin chains, for both integer and half-integer spins [1], much less has been achieved in the random case in particular for spin-1 chains. For the spin-1/2 random exchange Heisenberg antiferromagnetic chain (REHAC) theoretical advance [2, 3] has been motivated by experimental results mainly in organic materials [4]. The theory in this case gives a good description of the physical properties of these systems which are governed by low energy excitations [5]. Also recently an extensive study using a renormalization group approach has led to a deeper understanding of the zero temperature phase diagram of general spin-1/2 disordered quantum chains [3]. On the other hand the case of random, isotropic, spin-1 chains has only recently been addressed both experimentally [6, 7] and theoretically [0].

In this Communication we study a one-dimensional, spin-1, Heisenberg system with random exchange and biquadratic interactions. We use a real space renormalization group approach which has proved to be very sucessful for spin-1/2 quantum chains [2, 3]. We discuss the ground state properties and extend the approach to finite temperatures to obtain the thermodynamic properties.

We consider the general bilinear, biquadratic, spin-1 chain with $L$ spins described by the Hamiltonian

$$
H=\sum_{r=1}^{L-1} J_{r} \vec{S}_{r} \cdot \vec{S}_{r+1}-\sum_{r=1}^{L-1} D_{r}\left(\vec{S}_{r} \cdot \vec{S}_{r+1}\right)^{2}
$$


where $J_{r}$ and $D_{r}$ are random nearest-neighbor interactions with probability distributions $P_{J}\left(J_{r}\right)$ and $P_{D}\left(D_{r}\right)$ respectively and such that $0 \leq J_{r} \leq J$ and $0 \leq D_{r} \leq D$. The $\vec{S}_{r}$ are spin-1 operators and we are interested in the limit $L \rightarrow \infty$.

We study in this Communication the effect of disorder in two distinct situations:

1. The case where the biquadratic is the dominant interaction such that the cutoff $D>>J$. We recall that the ordered system with $D_{r}=D$ and furthermore $J_{r}=0$ corresponds to the so called $K B B$ point of the general phase diagram of the Hamiltonian given by Eq.1 [8]. At this point the purely biquadratic Hamiltonian can be solved exactly yielding a dimerized phase characterized by a two-fold degenerate ground state with a finite gap to the excited states.

2. The cutoff $J$ of the exchange interaction is much larger than that of the biquadratic couplings, i.e. $J>>D$. This corresponds to investigate the effect of disorder on the section of the phase diagram of the pure system where a Haldane gap occurs [1]. The ground state of the ordered system $\left(J_{r}=J, D_{r}=0\right)$ in this case is unique and also has a gap for excitations.

In order to deal with the Hamiltonian, Eq.1, we generalize, for the case of spin-1 and biquadratic interactions, a real space renormalization group 
method introduced by Ma, Dasgupta and Hu [2] to treat the spin-1/2 REHAC. The usefulness and limitations of this method have been intensively discussed by Fisher [3] in recent papers on quantum random spin- $1 / 2$ chains. In at least one of the situations we study here this approach converges to the exact solution of the random problem. The method consists of identifying the pair of spins with the strongest coupling in the random chain and eliminating it by considering the interaction with the neighboring spins of this pair as a perturbation. This procedure generates new couplings $J^{\prime}$ and $D^{\prime}$ between the two spins neighboring the eliminated pair and consequently the form of the distribution $P_{J}\left(J_{r}\right)$ and $P_{D}\left(D_{r}\right)$ are modified. One also gains a contribution to the free energy of the system from the eliminated pair. This process is then successively applied until a single pair of spins remains.

We start at zero temperature and consider the case of purely biquadratic interactions and no exchange. In the ordered case as mentionned above this corresponds to the so-called $K B B$ point [8] of the general phase diagram of the spin-1 chain given by the Hamiltonian of Eq.1. We assume a distribution of random biquadratic couplings and initially take $J_{r}=0$. Generalizing the procedure of Ma et al. [2] for this problem, we identify the pair of spins with the strongest biquadratic coupling. When we apply the elimination transformation to this strong coupled pair we find an effective coupling between the spins neighboring the eliminated pair which is is given by:

$$
D^{\prime}=\frac{2}{9} \frac{D_{1} D_{2}}{D}
$$


where $D_{1}$ and $D_{2}$ are the bonds connecting the strongest coupled pair of spins, with interaction strength $D$, to their neighbors. It is clear that in this case the successive application of the elimination transformation generates weaker and weaker couplings and the distribution of biquadratic interactions becomes peaked close to the origin as the cutoff $D$ decreases. The physical properties of the system are then dominated by low energy excitations and this new phase is very similar to the random singlet phase of the disordered spin-1/2 random antiferromagnetic chain [3]. Consequently disorder has a dramatic effect at the $K B B$ point since in the pure case the system has a dimerized phase with a gap for excitations and a short correlation length. The introduction of weak randomness on the biquadratic couplings gives rise to a new disordered phase with low energy excitations and long-range magnetic correlations. This behavior is universal in the sense that independently of the form of the initial distribution of couplings $P_{D}\left(D_{r}\right)$ this converges to a fixed point distribution, which is approximately described by a power law with a singularity at the origin [2]. Notice that the equation we find here renormalizes to weak coupling faster than in case of the spin-1/2 chain where the pre-factor of the recursion relation equivalent to Eq.2 is 1/2 [2]. We have found here a rather unusual situation where a phase with a gap and a short correlation length has become completely unstable and furthermore with long-range magnetic correlations due to the introduction of arbitrarily weak disorder [9]. 
We include now a weak random exchange interaction between nearest neighbor spins such that the cutoff $J<<D$. A generalization of the elimination transformation gives, for the new exchange and biquadratic couplings between the spins neighboring the eliminated pair, the following coupled relations

$$
\begin{gathered}
D^{\prime}=\frac{2}{9} \frac{D_{1} D_{2}}{D+J_{0}} \\
J^{\prime}=\frac{4}{3} \frac{J_{1} J_{2}}{\left(3 D+J_{0}\right)}
\end{gathered}
$$

where as before $D_{1}, D_{2}, J_{1}$ and $J_{2}$ are the bonds connecting the strongly coupled pair of spins, with interactions $D$ and $J_{0}$ between them, to their neighbors. Since $D>>J$ we expect, as before, that the renormalized distributions converge rapidly to a weak coupling situation, i.e., to develop more and more weight close to the origin as the cutoff decreases. Again the system attains a random singlet phase dominated by low energy excitations.

We remark that the recursion relations for the biquadratic interactions iterate to zero faster than that of the exchange couplings. It may happen that, depending on the starting distributions, the physics at sufficiently low energies will be dominated by the exchange interactions. In this case it is convenient to define the strongest coupled pair as that for which $3 D+J$, the energy to create an excited state for a pair, is larger.

For completeness we give now the expression for the energy associated with the eliminated pair and from which we can compute the total ground 
state energy

$$
\begin{gathered}
E^{\prime}=-2 J_{0}-4 D-\frac{4}{3}\left(D_{1}+D_{2}\right)-\frac{4}{3} \frac{J_{1}^{2}+J_{2}^{2}}{J_{0}+3 D} \\
-\frac{8}{27} \frac{D_{1}^{2}+D_{2}^{2}-D_{1} D_{2}}{J_{0}+D}
\end{gathered}
$$

We conclude this analysis of case 1 , i.e. $D>>J$, pointing out that we have obtained a new disordered phase for the random biquadratic spin-1 Hamiltonian which is similar to the random singlet phase of the spin- $1 / 2$ REHAC.

Case 2) We start with the situation where $D_{r}=0$. For the random exchange spin-1 chain, the elimination procedure yields the following relation for the new coupling between the spins neighboring the eliminated pair

$$
J^{\prime}=\frac{4}{3} \frac{J_{1} J_{2}}{J}
$$

We find here quite a distinct situation from the previous case, since this recursion relation appears to iterate to strong coupling. The generation of couplings which are larger than those eliminated would in fact invalidate the present approach based on perturbation theory. Contrary to the previous cases, now the results depend strongly on the form of the starting distributions. When we include the biquadratic interaction, for $D<<J$, the new recursion relations are similar to those obtained before, namely

$$
J^{\prime}=\frac{4}{3} \frac{J_{1} J_{2}}{\left(J+3 D_{0}\right)}
$$




$$
D^{\prime}=\frac{2}{9} \frac{D_{1} D_{2}}{J+D_{0}}
$$

for $D<<J$. It is clear that the biquadratic is an irrelevant interaction, in the renormalization group sense, for the random exchange spin-1 chain since the starting distribution $P_{D}\left(D_{r}\right)$ iterates to a distribution with most of it's weight at small coupling much faster than the exchange distribution. We notice that the biquadratic interactions reduce the new exchange couplings generated by the elimination transformation. Whether this is sufficient to guarantee the validity of the present approach will depend on the nature of the starting distributions and the values of the cutoffs.

In order to get a better understanding of the random exchange spin-1 chain, we have generalized the elimination transformation procedure to finite temperatures [7]. In the case $D_{r}=0$ we get after some lenghty calculations

$$
J^{\prime}=\frac{4}{3} \frac{J_{1} J_{2}}{J} W(\beta J)
$$

where $J_{1}$ and $J_{2}$ as before are the bonds connecting the strongly coupled pair of spins to their neighbors. We point out that this transformation does not generate new terms preserving the form of the original Heisenberg Hamiltonian. The free energy has a contribution from the eliminated pair which turns out to be,

$$
F^{\prime}=F_{0}-\frac{4}{3} \frac{J_{1}^{2}+J_{2}^{2}}{J} V(\beta J)
$$

with

$$
F_{0}=-2 J-\frac{1}{\beta} \operatorname{Ln}\left(1+3 e^{-\beta J}+5 e^{-3 \beta J)}\right)
$$


The quantities $\mathrm{W}(\mathrm{y})$ and $\mathrm{V}(\mathrm{y})$ are given by [7]:

$$
W(y)=\frac{1-(3 / 8) e^{-y}(1+y)-(5 / 8) e^{-3 y}(1+3 y)}{1+3 e^{-y}+5 e^{-3 y}}
$$

and

$$
V(y)=\frac{1-(3 / 8) e^{-y}(1-y)-(5 / 8) e^{-3 y}(1-3 y)}{1+3 e^{-y}+5 e^{-3 y}}
$$

Notice that for $k_{B} T>0.37 J$ the function $W(\beta J)<(3 / 4)$ and this guarantees the validity of the present approach for sufficiently high temperatures independent of the starting distribution.

For certain probability distributions, which we associate with the case of strong disorder, the successive elimination transformations give rise to weaker and weaker couplings as the cutoff $J$ decreases. In this case, for sufficiently small $J$, the distribution $P_{J}\left(J_{r}\right)$ becomes peaked at $J_{r} \approx 0$ and can be approximated by a power law [2, 7],

$$
P_{J}(x, J) \approx \frac{\alpha}{J} x^{-1+\alpha}
$$

where $x=J_{r} / J, J$ is the actual cutoff and the factor $(\alpha / J)$ arises from the normalization condition. This distribution leads to a low temperature behavior of the thermodynamic quantities which can be described by power laws [2, 4],

$$
F \propto T^{1+2 \alpha}, C_{v}=-T \frac{\partial^{2} F}{\partial F^{2}} \propto T^{\gamma_{c}}
$$

and

$$
\chi \propto T^{-1+\gamma_{s}}, m \propto H^{\gamma_{h}}
$$


where $\gamma_{c}$ is the specific heat exponent, $\gamma_{s}$ and $\gamma_{h}$ are related to the exponent $\alpha$ of $\mathrm{P}(\mathrm{x}, \mathrm{J})$ and of the free energy $F$. The susceptibility exponent is given, to a good approximation by, $\gamma_{s} \approx 2 \alpha$ and furthermore $\gamma_{h} \approx \gamma_{s}$ [2]. $H$ and $m$ are respectively the uniform external field and magnetization. Since the power law distribution is not the exact fixed point distribution for the elimination transformation, $\alpha$ and consequently $\gamma_{c}, \gamma_{s}$ and $\gamma_{h}$ depend on temperature and cutoff. For initial power law distributions, Eq.12, this dependence is very weak [2, [7].

The results contained in Eqs.13 have been explicitly verified for the strongly disordered cases of initial uniform, $P(K)=\Theta(1-K)$, and power law distributions (Eq.12) [7]. The free energy was obtained numerically from the accumulation point of Eq.8 for successive elimination transformations. Then Eqs.13 yield the exponent $\alpha$ and the specific heat [7]. For the same starting distribution and temperature range we found that the exponent $\alpha$ for the spin-1 chain is consistently larger than that for the spin-1/2. In fact, for any initial distribution which attains a power law form whenever the cutoff is sufficiently small, we can show that the exponent $\alpha$ decreases more rapidly when the cutoff is further reduced for $S=1 / 2$ than for $S=1$ [0]. These theoretical results are in agreement with experiments in the strongly disordered, one-dimensional, spin-1 system $\mathrm{MgVOBO}_{3}$ which shows power law behavior of the susceptibility in a wide temperature range [7] although less singular than in the isostructural spin-1/2 material $\mathrm{MgTiOBO}_{3}$ [5]. 
The elimination transformation for $S=1$, Eq.7 or Eq.5, applies for strongly disordered chains, which we generically define as those characterized by initial distributions which yield thermodynamic properties at low temperatures as described by Eqs.13 and 14. For these distributions couplings larger than the one eliminated are generated with negligeable probability and the cutoff decreases rapidly enough to yield meaningful thermodynamic behavior as in the cases of uniform and power law distributions. Otherwise our results represent a good approximation for the high temperature behavior of disordered spin-1 chains.

We have extended the elimination transformation method for a quantum Heisenberg chain with arbitrary spin. We find the following recursion relation

$$
J^{\prime}=\frac{2}{3} S(S+1) \frac{J_{1} J_{2}}{J}
$$

This result shows that perturbation theory fails for large spins since the excited states become nearly degenerate with the ground state. The critical value of $S$ is between $S=1 / 2$ and $S=1$ so that a universal random singlet type of phase in the Heisenberg chain with exchange interactions only is guaranteed for spin- $1 / 2$. In this case the quantum fluctuations are sufficiently strong and the renormalization group equations yield a flow to a fixed point universal distribution singular at the origin.

In conclusion we have generalized an elimination transformation procedure to study the effect of disorder on quantum antiferromagnetic chains with exchange and biquadratic interactions. In case the biquadratic inter- 
action dominates, we find a new disordered phase for spin-1 chains which is essentially a random singlet phase. We find that weak disorder destroys the dimerized phase of the ordered system giving rise to low energy excitations and long range correlations. In the Haldane region of the phase diagram of the pure chain our results indicate that weak disorder has not a profound effect although the present approach fails in this case at least at low temperatures. However for starting distribuitons which are strongly disordered we obtain a thermodynamic behavior which is similar to the random singlet phase of disordered spin-1/2 REHAC but with weaker singularities.

\section{Acknowledgements}

We would like to thank Prof. E. Muller-Hartmann and Prof. J.C.Fernandes for invaluable discussions. 


\section{References}

[1] Ian Affleck, Rev. in Math. Phys., 6 , No. 5a, 887 (1994); F.D.M.Haldane, Phys. Rev. Lett. 50 , 1153 (1983).

[2] S.K.Ma, C. Dasgupta and C. K. Hu, Phys.Rev.Lett. 43 , 1434 (1979);

C. Dasgupta and S. K. Ma, Phys.Rev.B22 , 1305 (1980).

[3] D.S.Fisher, Phys.Rev. B50 , 3799 (1994);ibid Phys. Rev. B51 , 6411 (1995).

[4] W.G.Clark in Physics in One Dimension, pag. 289, edited by J. Bernasconi and T. Schneider, Springer Series in Solid State Science, Springer-Verlag, 1981.

[5] J.C.Fernandes et al., Phys.Rev.B50 , 16754 (1994).

[6] J.F.DiTusa et al., Phys.Rev.Lett. 73 , 1857 (1994).

[7] M. A. Continentino, J. C. Fernandes, R. B. Guimarães, B. Boechat, H. A. Borges, J. V. Valarelli, E. Haanappel, A. Lacerda and P. R. J. Silva, to be published in Philosphical Magazine.

[8] A. Klumper, Europhys. Lett.9 , 815 (1989). M. T. Batchelor and M. N. Barber, Phys.Rev. B40 , 4621 (1989);ibid J.Phys. A23 L15 (1990).

[9] R. A. Hyman, Kun Yang, R. N. Bhatt and S. M. Girvin, Cond-Mat pre-print 9505095. 